\title{
CONTROL DE UN MANIPULADOR ANTROPOMÓRFICO POR MEDIO DE UN DISPOSITIVO DE INMERSIÓN
}

\author{
Ricardo A. Castillo ${ }^{1}$, Carlos D. Velásquez ${ }^{1}$, Óscar F. Avilés ${ }^{2}$, Iván A. Olier ${ }^{3}$ \\ (1) Ingeniero mecatrónico Universidad Militar Nueva Granada ricard333@hotmail.com \\ (1) Ingeniero mecatrónico Universidad Militar Nueva Granada carlos_verne21@hotmail.com \\ (2) Ingeniero electrónico. Especialista en instrumentación electrónica, candidato a MSc Universidad Tecnológica de Pereira. Profesor \\ Universidad Militar Nueva Granada. oaviles@umng.edu.co \\ (3)Ingeniero mecánico, candidato a PhD Universidad de Barcelona iaolier@1si.upc.es
}

Fecha de recepción: 7 de junio de 2004

Fecha de aprobación: 7 de julio de 2004

Resumen-Este trabajo consta de 3 etapas: a) Construcción: dispositivo de inmersión, b) Desarrollo: software de simulación con $R V$, c) Diseño: sistema electrónico de adquisición y envío de datos. El dispositivo de inmersión captura los movimientos del brazo del operario, por medio de sensores; las señales suministradas por esto, son procesadas por un PIC, y enviadas al PC, para ser leídas por el software, que simula con realidad virtual el movimiento del manipulador UMNG-1. Finalmente, teniendo en cuenta la(s) posición(es) final deseada del efector final, es entregado un código, que enviado al controlador YASKAWA-SMC 2000, posibilita el movimiento real del UMNG-1, para llevar a cabo tareas de difícil ejecución.

Palabras clave: RV, PIC, manipulador, YASKAWA, SMC 2000.

\section{INTRODUCCIÓN}

$\mathrm{L}$ a realización de prototipos de brazos móviles para la ejecución de diferentes tareas, ha buscado que actividades que antes eran realizadas por individuos, en condiciones difíciles y en entornos de trabajo cada vez más variados y peligrosos para la integridad física o la vida de la persona, sean realizadas por máquinas. específicamente por brazos $\mathrm{o}$ manipuladores teledirigidos. Pensando en esto surge la necesidad de dotar a la Universidad Militar Nueva Granada de un sistema de inmersión para controlar el robot UMNG-1, posibilitando el movimiento de este manipulador con base en un sistema de telerrobótica flexible y muy fácil de utilizar para cualquier persona.

Con la realización de este proyecto se desea programar el comportamiento de los 6 grados de libertad (DOF) del manipulador, controlando la posición de cada una de las articulaciones de este robot. En el armazón sensorial o dispositivo de inmersión, hecho de acrílico, se ubican sensores con los cuales se leen los ángulos de: tronco, antebrazo, brazo y muñeca del operario, y luego con base en estas posiciones se programaran los movimientos del manipulador sin necesidad de cálculos cinemáticos directos e inversos; luego, al grabar estas

posiciones en un PC, es posible programar el robot para tareas repetitivas.

El código generado permitirá que el manipulador UMNG-1 (Figura 1) sea utilizado en tareas tales como posicionamiento de materiales pesados y manipulación de sustancias químicas peligrosas, manipulación de objetos ubicados en posiciones difíciles de alcanzar para un brazo humano o en lugares muy distantes, la soldadura de piezas especiales, posicionamiento exacto de instrumentos quirúrgicos etc.; en la generación del código el operario no tendrá nada que ver con operaciones matemáticas de control de movimiento, sino que solamente dará instrucciones al manipulador con el movimiento de su brazo.

\section{MÉTODOS}

MANIPULADOR UMNG-1

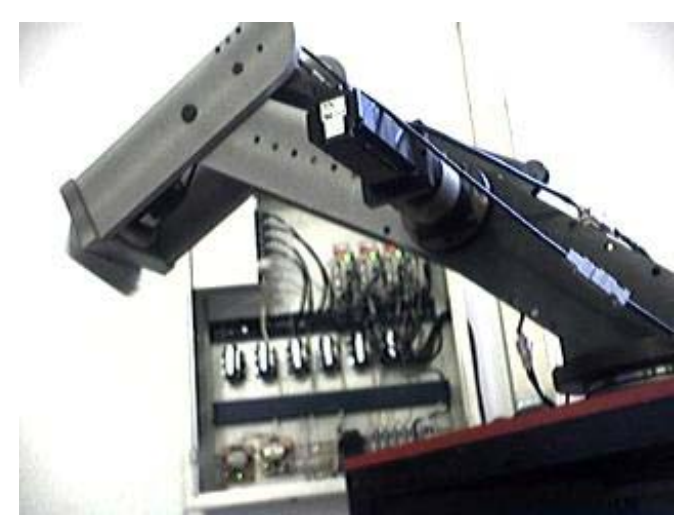

Fig. 1. Manipulador $\boldsymbol{U} \boldsymbol{M N G}$-1. Diseñado y desarrollado en la Universidad Militar Nueva Granada. Bogotá, Colombia 
El UMNG-1 es un manipulador antropomórfico de 6 DOF (grados de libertad) rotacionales, cuyo diseño lo asemeja bastante a un brazo humano con sus 6 grados básicos de libertad [2].

Tabla 1. Parámetros de desempeño del manipulador UMNG-1

\begin{tabular}{|c|c|}
\hline Carga máxima & $6 \mathrm{~kg}$ \\
\hline Alcance & $1.5 \mathrm{~m}$ \\
\hline Velocidad máxima & $1 \mathrm{~m} / \mathrm{s}$ \\
\hline Precisión & $+-3 \mathrm{~mm}$ \\
\hline
\end{tabular}

\section{A. Cinemática directa del manipulador UMNG-1}

Para lograr una correcta determinación de la posición final del último elemento del robot, se hizo uso de la cinemática directa; al considerar sus 6 grados de libertad, se obtendrá que la posición y orientación del eslabón final vendrá dada por la matriz de transformación total $T_{0}^{6}$, que resulta de la multiplicación de las matrices de transformación que describen la posición de eslabones contiguos, como se describe a continuación [1]:

$$
T_{0}^{n}=T_{0}^{1} T_{1}^{2} T_{2}^{3} T_{3}^{4} T_{4}^{5} T_{5}^{6}
$$

Para describir la relación que existe entre 2 elementos contiguos, se hace uso de Denavit-Hartemberg (D-H) (Figura 2), en donde $\theta_{i}, a_{i}, d_{i}, \alpha_{i}$ son los parámetros de Denavit-Hartemberg (D-H) del eslabón [1].

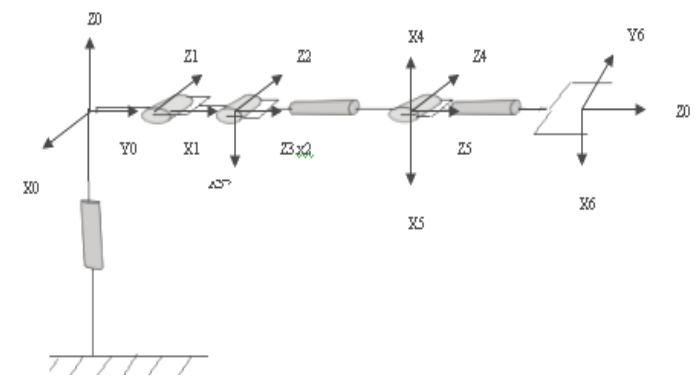

Fig. 2. Representación esquemática del manipulador UMNG-1, sobre el cual se han ubicado los sistemas coordenados

A partir de los parámetros estructurales del robot UMNG-1(Tabla 2) y teniendo como base la ecuación de transformación homogénea,

$$
T_{i-1}^{i}=\left[\begin{array}{cccc}
C \theta_{i} & -C \alpha_{i} S \theta_{i} & S \alpha_{i} S \theta_{i} & a_{i} C \theta_{i} \\
S \theta_{i} & C \alpha_{i} C \theta_{i} & -S \alpha_{i} C \theta_{i} & a_{i} S \theta_{i} \\
0 & S \alpha_{i} & C \alpha_{i} & d_{i} \\
0 & 0 & 0 & 1
\end{array}\right]
$$

se obtiene la ecuación de transformación de cada eslabón con respecto al anterior, remplazando los parámetros correspondientes, y por lo tanto también se encuentra la matriz de transformación total $T_{0}^{6}$, que describe la posición del efector final del UMNG-1 respecto de su base, al realizar la multiplicación de matrices [1]:

$$
T_{0}^{n}=T_{0}^{1} T_{1}^{2} \ldots . T_{n-1}^{n}
$$

Donde $\mathrm{n}$ es el número de grados de libertad, para nuestro caso $\mathrm{n}=6$.

Tabla 2. Parámetros estructurales del manipulador UMNG-1 [4]

\begin{tabular}{|c|c|c|c|c|c|}
\hline Artic. & $\begin{array}{c}\Theta i \\
(\mathrm{rad})\end{array}$ & $\begin{array}{c}\alpha \mathrm{i} \\
(\mathrm{rad})\end{array}$ & $\begin{array}{c}\text { ai } \\
(\mathrm{mm})\end{array}$ & $\begin{array}{c}\mathrm{di} \\
(\mathrm{mm})\end{array}$ & Rango \\
\hline $\mathbf{1}$ & $\boldsymbol{\pi} / \mathbf{2}$ & $-\boldsymbol{\pi} / \mathbf{2}$ & $\mathbf{2 0 0}$ & $\mathbf{0}$ & $\mathbf{- 1 6 0} \leq \boldsymbol{\theta 1} \leq \mathbf{1 6 0}$ \\
\hline $\mathbf{2}$ & $\mathbf{0}$ & $\mathbf{0}$ & $\mathbf{4 8 0}$ & $\mathbf{0}$ & $\mathbf{- 1 5 0} \leq \boldsymbol{\theta 2} \leq \mathbf{4 5}$ \\
\hline $\mathbf{3}$ & $\boldsymbol{\pi} / \mathbf{2}$ & $\boldsymbol{\pi} / \mathbf{2}$ & $\mathbf{0}$ & $\mathbf{0}$ & $\mathbf{1 3 5} \leq \boldsymbol{\theta 3} \leq \mathbf{2 2 5}$ \\
\hline $\mathbf{4}$ & $\boldsymbol{\pi}$ & $\boldsymbol{\pi} / \mathbf{2}$ & $\mathbf{0}$ & $\mathbf{5 5 5}$ & $\mathbf{- 1 8 0} \leq \boldsymbol{\theta 4} \leq \mathbf{1 8 0}$ \\
\hline $\mathbf{5}$ & $\boldsymbol{\pi}$ & $\boldsymbol{\pi} / \mathbf{2}$ & $\mathbf{0}$ & $\mathbf{0}$ & $\mathbf{9 0} \leq \boldsymbol{\theta 5} \leq \mathbf{2 7 0}$ \\
\hline $\mathbf{6}$ & $\mathbf{0}$ & $\mathbf{0}$ & $\mathbf{0}$ & $\mathbf{2 1 6}$ & $\mathbf{0} \leq \boldsymbol{\theta 6} \leq \mathbf{3 6 0}$ \\
\hline
\end{tabular}

\section{B. Similitudes entre el manipulador UMNG-1 y el brazo humano}

El diseño del manipulador UMNG-1 se asemeja bastante a un brazo humano (Figura 3) con sus 6 grados básicos de libertad; la configuración geométrica normal del brazo humano es igual a la configuración del manipulador UMNG-1 luego de ser girado 180 grados (Figura 4).

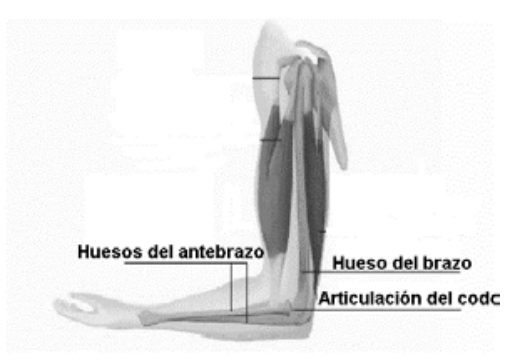

Fig. 3. Vista del brazo humano en una configuración normal [3] 


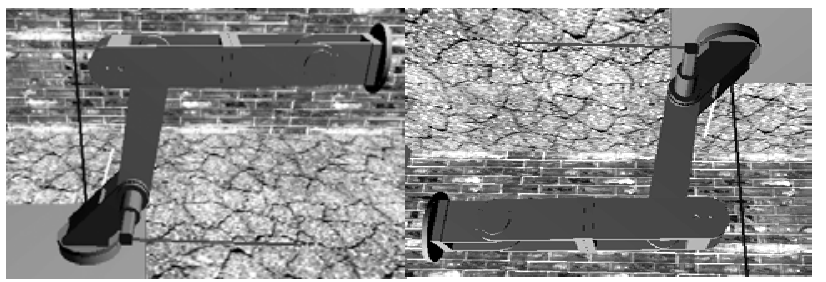

(a)

(b)

Fig. 4. (a) Manipulador UMNG-1, una configuración normal; (b) Manipulador UMNG-1 girado a 180 grados.

\section{Correspondencia de las articulaciones-elementos entre el Robot UMNG-1 y el dispositivo de inmersión}

Con el fin de diseñar el sistema mecánico del dispositivo de inmersión, se tuvo en cuenta que este permitiera el ajuste al brazo del operario, de forma que se pudieran capturar el movimiento de giro de cada una de las articulaciones del miembro superior (4 DOF), el accionamiento del efector final (1 DOF), así como el giro de la articulación del tronco del operario (1 DOF). Como se puede observar, se necesita leer la totalidad de los grados de libertad, cada uno de los cuales controlará a su vez 1 solo grado de libertad del manipulador UMNG-1; existe una correspondencia 1 a 1 entre articulaciones (Tabla 3). Es importante destacar que la correspondencia entre las articulaciones del dispositivo de inmersión se ha hecho lo más cercana posible a las articulaciones del manipulador, de manera que podamos asemejar el movimiento del UMNG- 1 al de un brazo humano, y por lo tanto controlarlo con el movimiento de este (mediante el dispositivo de inmersión) (Tabla 4).

Tabla 3. Equivalencias entre los eslabones del UMNG-1 y el brazo humano

\begin{tabular}{|l|l|}
\hline \multicolumn{1}{|c|}{$\begin{array}{c}\text { ARTICULACIÓN DEL } \\
\text { ROBOT }\end{array}$} & $\begin{array}{c}\text { CORRESPONDENCIA CON } \\
\text { LAS ARTICULACIONES } \\
\text { HUMANAS }\end{array}$ \\
\hline $\begin{array}{l}\text { Articulación 1 } \\
\text { (Giro de la base) }\end{array}$ & $\begin{array}{l}\text { Giro del tronco del operario (giro } \\
\text { cintura) }\end{array}$ \\
\hline Articulación 2 & Giro del hombro del operario \\
\hline Articulación 3 & Giro del codo del operario \\
\hline Articulación 4 & Giro del antebrazo del operario \\
\hline Articulación 5 & Giro de la muñeca del operario \\
\hline $\begin{array}{l}\text { Articulación 6 (efector } \\
\text { final) }\end{array}$ & $\begin{array}{l}\text { Accionamiento de la mano del } \\
\text { operario }\end{array}$ \\
\hline
\end{tabular}

También se pensó en realizar una correspondencia entre cada una de las partes del miembro superior y los eslabones que conforman al manipulado

Tabla 4. Equivalencias entre los eslabones del UMNG-1 y el brazo humano.

\begin{tabular}{c|c} 
COMPONENTE & CORRESPONDENCIA CON EL \\
ROBOT UMNG-1 & BRAZO HUMANO
\end{tabular}

\begin{tabular}{|l|l|}
\hline Mesa de soporte del robot & $\begin{array}{l}\text { Parte debajo de la cintura del } \\
\text { operario }\end{array}$ \\
\hline Base o eslabón 1 del robot & Tronco del operario \\
\hline Eslabón 2 del robot & Brazo del operario \\
\hline Eslabón 3 del robot & Antebrazo del operario \\
\hline Eslabón 4 del robot & Antebrazo del operario \\
\hline Eslabón 5 del robot & Mano del operario \\
\hline $\begin{array}{l}\text { Eslabón 6 del robot } \\
\text { (efector final) }\end{array}$ & Dedos del operario \\
\hline
\end{tabular}

Con el fin de construir un dispositivo de inmersión, que simulará en su configuración tanto de eslabones como de articulaciones al brazo human, el dispositivo de inmersión, en la parte correspondiente al miembro superior se compone de 5 partes diseñadas para ajustarse de manera cómoda al brazo del usuario ya que con el antebrazo humano es posible controlar 2 grados de libertad del robot mediante : giro del codo y giro del antebrazo sobre un eje paralelo a este; la pieza del antebrazo es un conjunto constituido por 2 partes (tabla 5).

Tabla 5. Equivalencias entre el brazo humano y el sistema de inmersión

\begin{tabular}{|c|c|}
\hline $\begin{array}{c}\text { PARTE DEL BRAZO } \\
\text { HUMANO }\end{array}$ & $\begin{array}{c}\text { PARTE DEL SISTEMA DE } \\
\text { INMERSIÓN }\end{array}$ \\
\hline $\begin{array}{c}\text { Parte bajo la cintura del } \\
\text { operario }\end{array}$ & $\begin{array}{c}\text { Base inferior del dispositivo de } \\
\text { inmersión }\end{array}$ \\
\hline Tronco del operario & Base superior del dispositivo \\
\hline Brazo del operario & $\begin{array}{c}\text { Pieza del hombro y conjunto pieza } \\
\text { del brazo en el dispositivo }\end{array}$ \\
\hline Antebrazo del operario & $\begin{array}{c}\text { Conjunto pieza del antebrazo } \\
\text { (2 partes) }\end{array}$ \\
\hline Mano del operario & Pieza de la mano del dispositivo \\
\hline Dedos del operario & $\begin{array}{c}\text { Controles digitales de la } \\
\text { empuñadura en la pieza de la mano } \\
\text { (botones) }\end{array}$ \\
\hline
\end{tabular}

\section{Funcionamiento general de los sistemas implementados}

- El sistema de inmersión permitirá capturar los movimientos del brazo del operario, lo cual se realiza por medio de goniómetros (sensores de posición angular).

- Cada una de las señales suministradas por estos sensores es enviada a un PIC, en el cual es procesada para su posterior envió al PC.

- Por medio del Driver de comunicación y utilizando un protocolo de intercambio de datos con el puerto paralelo, las señales son leídas en el software de simulación.

- Con base en las señales provenientes de los sensores, es generada una simulación en realidad virtual del movimiento de robot UMNG-1, teniendo en cuenta la posición de cada una de sus articulaciones.

- Finalmente y teniendo en cuenta la(s) posición(es) final deseada del ultimo elemento del robot, es generado el código que debe ser enviado al controlador YASKAWA-SMC 2000 con el cual se posibilita el movimiento real del manipulador: 


\begin{tabular}{|c|c|c|c|}
\hline & & & GENERACION \\
\hline SI & $\begin{array}{l}\text { SISTEMA DE } \\
\text { TRATAMIENTO }\end{array}$ & SOFTWARE DE & $\mathrm{DEL}$ \\
\hline INMERSI & DE SEN̈AL Y & $\begin{array}{c}\text { SIMULACION } \\
\text { RV }\end{array}$ & DE CONTROL \\
\hline
\end{tabular}

Fig. 6. Esquema general del sistema implementado.

\section{Diseño y construcción del dispositivo de inmersión}

Este dispositivo está diseñado para sensar la posición angular de las articulaciones del brazo de un operario y enviarlas al PC con el fin de efectuar una simulación ON-LINE del funcionamiento del manipulador. Las piezas del dispositivo de inmersión (Figura 7) se encuentran en su totalidad fabricadas de acrílico.

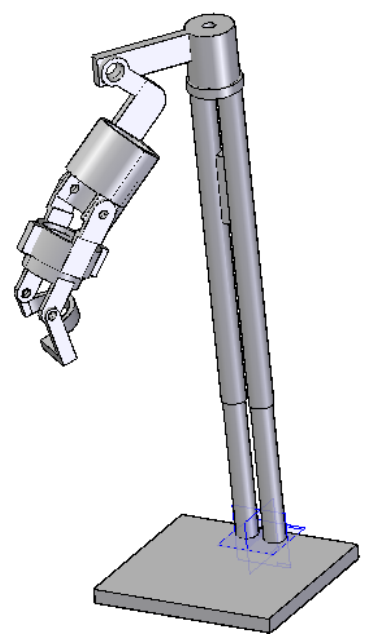

Fig. 7. Dispositivo de inmersión completo

\section{Piezas correspondientes al brazo en el dispositivo de inmersión.}

\section{Pieza del hombro}

La primera pieza está diseñada para leer el movimiento en un solo eje, el eje del hombro del operario.

\section{Pieza del brazo}

La pieza del brazo junto con la pieza del hombro, está diseñada para leer el movimiento angular del hombro. Esta pieza está diseñada de una manera ergonómica, de modo que pueda acomodarse fácilmente a cualquier tipo de brazo; el ajuste de esta parte se da tanto en el grosor del brazo como en la longitud de este.

\section{Pieza del antebrazo}

La pieza 3 en realidad es un conjunto de 2 piezas, acopladas de tal forma que se permite el movimiento entre ellas con 1 grado de libertad (1 DOF rotacional): giro relativo de las piezas a lo largo del eje del antebrazo

\section{Pieza de la mano}

Esta pieza, además de posibilitar el giro de la articulación de la muñeca, facilita el movimiento total de la articulación del antebrazo, ya que solo si el operario sujeta con su mano esta pieza, se podrán detectar de forma eficaz los 2 movimientos de la muñeca:

- Giro en un eje paralelo al antebrazo

- Giro en un eje perpendicular al antebrazo

\section{Sensores}

Los sensores que se utilizan para capturar los movimientos del dispositivo de inmersión son potenciómetros lineales de precisión de una sola vuelta (Figura 8). El sensor es colocado en el eje de rotación de cada articulación. Ya que los sensores cambian su valor resistivo cuando su eje central rota, la implementación de estos en el dispositivo es óptima, teniendo en cuenta que el parámetro que se desea medir es el giro de cada articulación.

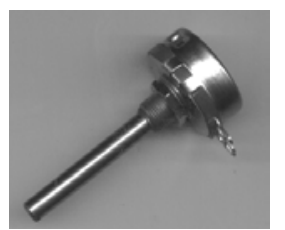

Fig. 8. Sensor utilizado en el dispositivo de inmersión

\section{Software de simulación RV del manipulador}

El software de control permite la visualización ON-LINE del movimiento del manipulador midiendo las posiciones angulares de cada una de las articulaciones del dispositivo de inmersión y relacionándolas con las articulaciones del robot. Además, permite obtener una posición deseada del UMNG-1, la cual es grabada y traducida al lenguaje del controlador YASKAWA SMC-2000, para luego generar un archivo con las instrucciones necesarias para el movimiento real del manipulador UMNG-1

Dado que el software (Figura 10) está escrito enteramente en $\mathrm{C}++$ Builder 6.0, es posible establecer un protocolo de comunicación entre el PC y el dispositivo de inmersión por medio del puerto paralelo, utilizando la Interfaz EPP (Enhanced Parallel Port). Entre las características más importantes del software tenemos:

\section{Recolección de las señales enviadas desde los sensores en el dispositivo de inmersión}

El software es diseñado para leer de manera ordenada cada una de las 6 señales enviadas por el PIC, correspondientes a cada uno de los 6 sensores, ubicados en las articulaciones del dispositivo de inmersión.

\section{Representación virtual del manipulador}


Es una de las características más sobresalientes y llamativas del software ya que permite ver al manipulador UMNG-1 de una manera escalizada y correcta (Figura 9). Además, posibilita observar el movimiento independiente de cada uno de los eslabones que lo conforman, mientras se está utilizando el dispositivo de inmersión

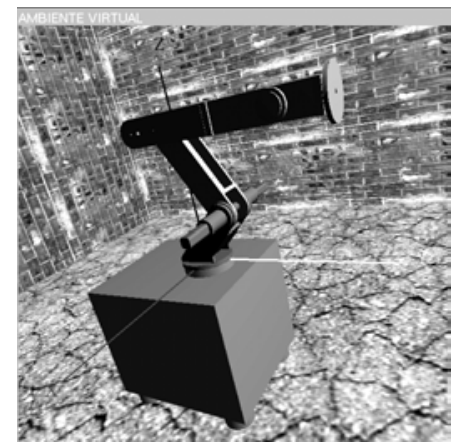

Fig. 9. Vista del manipulador dentro del software de simulación

\section{Información continua y en tiempo real de las coordenadas del efector final del manipulador}

Mediante el cálculo de la cinemática, teniendo en cuenta el valor angular en cada instante de cada una de las articulaciones del manipulador, se puede leer la posición exacta de su efector final, con el fin de controlar la ubicación a la cual deseamos mover el robot para que desempeñe una determinada tarea.

Relación entre los desplazamientos angulares de las articulaciones del dispositivo de inmersión y las articulaciones del manipulador

Los rangos totales de movimiento de las articulaciones del robot se acoplan a los rangos totales de movimiento de las articulaciones del brazo del usuario mediante la inserción de factores de corrección.

Generación del código necesario para el controlador YASKAWA SMC-2000

Cuando el operario, utilizando el dispositivo de inmersión, ha llevado o posicionado el efector final del manipulador en la ubicación deseada, entonces accionando una opción del menú desplegable, se generaran 2 archivos los cuales contendrán las instrucciones para el controlador del robot.

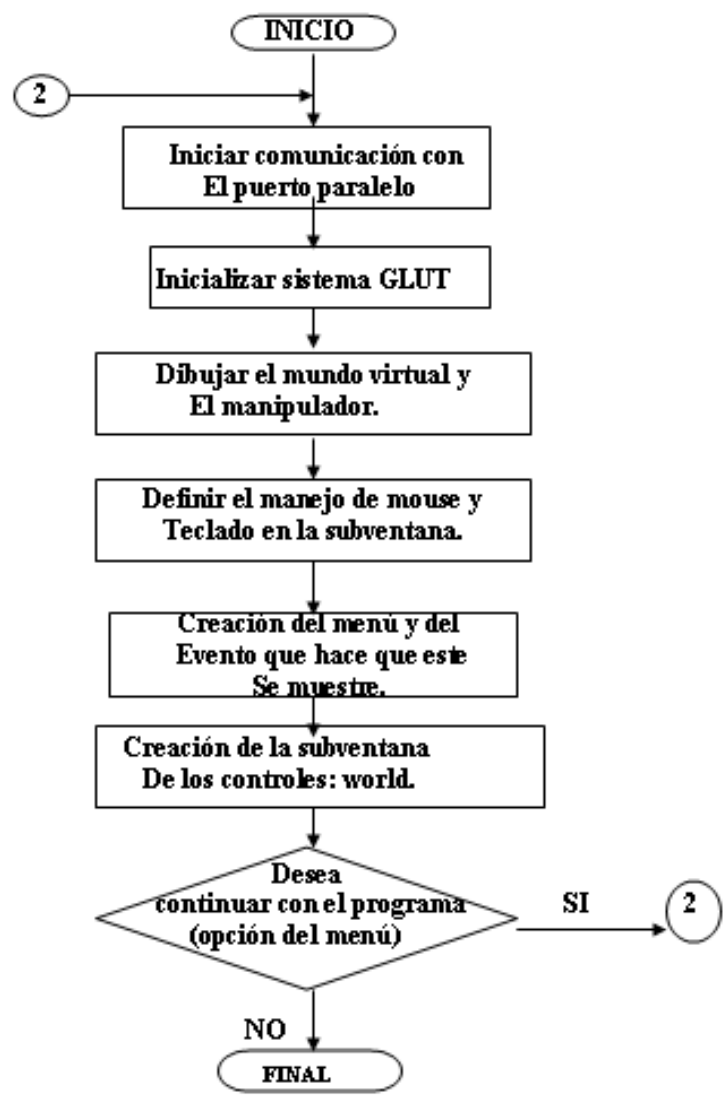

Fig. 10. Diagrama de flujo del software de simulación.

\section{DRIVER Y CIRCUITO DE COMUNICACIÓN CON EL}

PC

Está diseñado para leer las señales obtenidas de los sensores en el dispositivo de inmersión y enviarlas al PC.

\section{Software en el Pic para capturar señales del dispositivo de inmersión}

Se decidió usar un microprocesador PIC-16F877-04, debido a su fácil manejo, a la cantidad de puertos que presenta y al número de entradas análogas que puede manejar. El programa en este PIC (Figura 11) fue hecho en su totalidad en lenguaje Mplab (Assembler); en este caso se utilizan el puerto A y el E como entradas análogas y digitales, y el puerto $\mathrm{C}$ como salida digital, la cual es conectada al puerto paralelo del computador. 


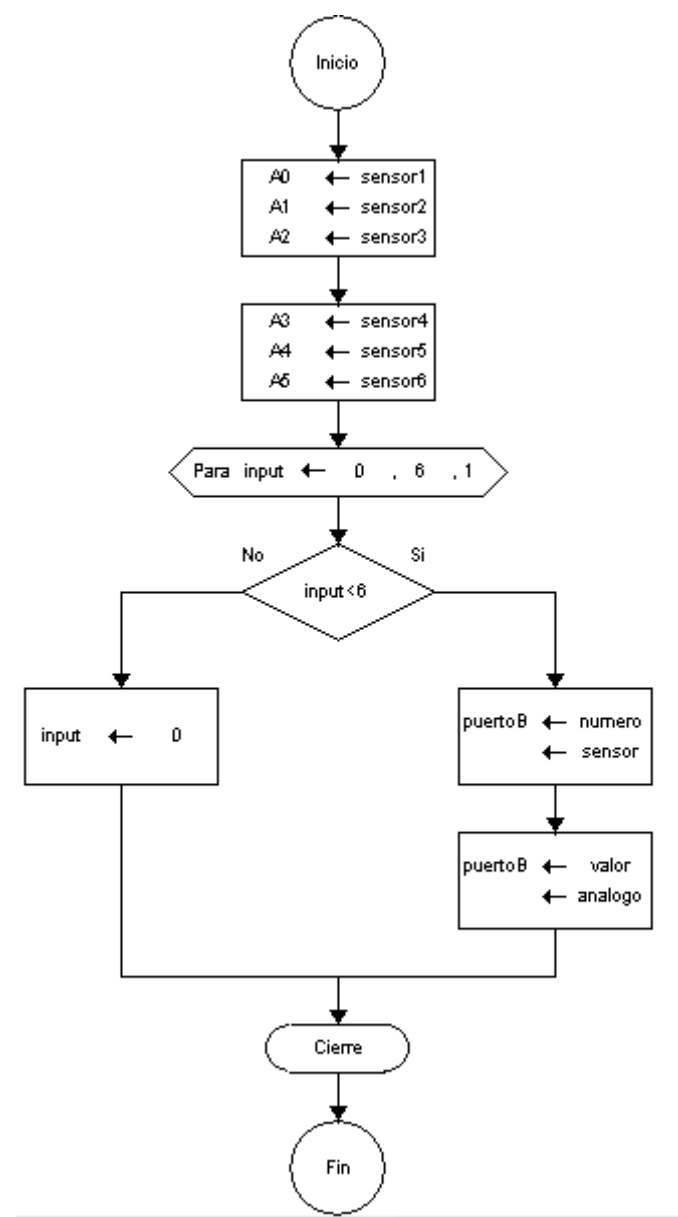

Fig. 11. Diagrama de flujo del programa del PIC

\section{CONCLUSIONES}

- Se lograron detectar con eficacia los movimientos de cada una de las articulaciones del brazo de un operario, pudiendo sensar con potenciómetros lineales los ángulos de giro de cada una de estas.

- Se encontraron parámetros de ergonomía y comodidad para el operario, de manera que este no presente dificultades en la utilización del dispositivo de inmersión.

- Se identificó el PIC 16F877 como un dispositivo adecuado para la implementación del driver de comunicación entre el dispositivo de inmersión y el PC; por ello, este se utilizo con el fin de detectar y multiplexar cada una de las señales enviadas por los sensores, para luego transmitirlas al PC en forma ordenada.

- Se encontró que era posible el diseño idóneo del software de simulación del funcionamiento del manipulador en $\mathrm{C}++$ Builder, utilizando librerías gráficas de OpenGL. Con estas herramientas se posibilitó que este software ofreciera: posibilidades de paneo, rotación y acercamiento en el mundo virtual, información de la posición del efector final del UMNG-1; además, detección de los movimientos de cada articulación en un rango permitido, con información de sobrepaso de rango y colisiones.

\section{REFERENCIAS BIBLIOGRÁFICAS}

[1] Jiménez, Antonio. Fundamentos de la Robótica. Barcelona, noviembre de 1996, p. 2-14., 93-100

[2] Casa Editorial El Tiempo. Enciclopedia Visual Seres Vivos. Bogotá-Colombia. 1992, p. 24-25.

[3] www.ergonomialcapitulo 2_aptitud fisica.htm

\section{BIBLIOGRAFÍA}

- Jiménez, Antonio. Fundamentos de Robótica. Barcelona, noviembre 1996, págs. 2-14., 93-100

- Universidad Militar "Nueva Granada". 1er. Seminario Internacional de Mecatrónica, Realidad Virtual y Sistemas Avanzados de Manufactura. Bogotá-Colombia. 2002, págs. 247-288

- Jaspe, Alberto. Dorado, Julián. Una aproximación a OpenGL. 2003, págs. 4-8,12

- Olier, Iván. Revista Ciencia e Ingeniería Neogranadina. Centro de Investigaciones de la Facultad de Ingeniería. Julio 2001, págs. 10-14

- Casa Editorial El Tiempo. Enciclopedia Visual Seres Vivos. Bogotá-Colombia. 1992, p. 24-25.

- $\quad$ MICROCHIP, PIC16F877-04, 40- pin, 8- bit CMOS FLASH Microcontroller. Chandler. Microchip Tecnology Inc. 2001, págs. 1,6,14.

- YASKAWA. SMC-2000 Multi-Axis Motion Controller, User's Guide Versión 3.1. págs. 9-12, 39, 49-53.

- Catálogo Multimedia de SKF. USA. 1998

- User manual. Deneb IGRIP. 2000

- $\quad$ Sedra, Adel /SMITH, Kenneth. Circuitos Microelectrónicas, Oxford University Press, USA, 1998, págs. 1058-1069.

- $\quad$ www.chi.itesm.mx/ cim/robind/robotic2.

- www.activamente.com.mx/vrml/.

- $\quad$ www.decom.es/area.php?cod=1

- www.mecc.polimi.it/ rovetta/robotica/ HTML/studentimangia/Telerobotica.htm

- www.nied.unicamp.br/ siros/doc/ artigo_wie2003_final.PDF

- $\quad$ www.esi2.us.es/ aollero/robotica.htm

- www.Ambientes Virtuales en Intranet.htm

- www.monografias.com/Realidad Virtual.htm

- www.cpr2valladolid.com/tecno/cyr 01/robotica/industrial - www.ergonomialcapitulo 2_aptitud fisica.htm

- $\quad$ www.nehe.gamedev.net. Clases Particulares de OpenGL

- $\quad$ www.linux-cgi.tccw.wku.edu/ graphics/SetupOpenGLB

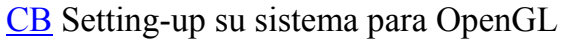

